\title{
Aproximación al perfil físico y antropométrico en vela ligera, en regatistas de la clase Optimist. Estudio de casos
}

\section{Approximation to the physical and anthropometric profile in dinghy sailing, in the Optimist class sailors. Study of cases}

\author{
Virginia Tejada-Medina y Javier Ventaja-Cruz
}

Universidad de Granada. (España).

\begin{abstract}
Resumen: El propósito de este estudio es determinar el perfil físico del regatista de Optimist, mediante la realización de una serie de pruebas físicas (SJ, flexibilidad y dinamometría manual) y de composición corporal (peso, talla, pliegues y perímetros musculares), describiendo el comportamiento de los indicadores seleccionados y valorando el carácter predictivo de los mismos, para una posible justificación de la existencia de un talento deportivo en Optimist. Se han estudiado a 3 regatistas, que ocupan los tres primeros puestos del ranking provincial de la Ciudad Autónoma de Melilla. Se utilizó un diseńo descriptivo correlacional. Los regatistas presentaron los siguientes valores descriptivos de composición corporal: altura $(149,6 \pm 2,16$ $\mathrm{cm})$, peso $(35,6 \pm 3,67 \mathrm{~kg})$, IMC $(15,93 \pm 2,04)$ y $\% \mathrm{GC}(7,86 \pm 5,31)$. En cuanto a las características funcionales, los valores fueron: $\mathrm{SJ}$ hcg $(22,53 \pm 1,68$ $\mathrm{cm})$, SJ tv $(428,6 \pm 16,28 \mathrm{sg})$, flexibilidad $(31,67 \pm 1,15)$ y dinamometría D-I $(23,83 \pm 2,02 ; 20,33 \pm 1,25)$. Los resultados no muestran una relación significativa entre las variables de composición corporal y las pruebas físicas realizadas.
\end{abstract}

Palabras clave: Talentos, clase Optimist, antropometría, composición corporal, regatista.
Abstract: The aim of this study is to determine the physical profile of the Optimist sailor, by performing a series of physical tests (SJ, flexibility and manual dynamometry) and body composition (weight, height, skinfolds and muscle perimeters), describing the behavior of the selected indicators and assessing the predictive nature of them, for a possible justification of the existence of a sports talent in Dinghy Optimist. Three sailors have been studied, occupying the first three positions of the provincial ranking of the Autonomous City of Melilla. A descriptive correlational design was used. The sailors presented the following descriptive values of body composition: height $(149.6 \pm 2.16 \mathrm{~cm})$, weight $(35.6 \pm 3.67 \mathrm{~kg})$, BMI $(15.93 \pm 2.04)$ and $\%$ GC $(7,86 \pm 5.31)$. Regarding the functional characteristics, the values were: SJ hcg $(22.53 \pm 1.68 \mathrm{~cm})$, SJ tv $(428.6 \pm 16.28 \mathrm{sg})$, flexibility $(31.67 \pm 1,15)$ and DI dynamometry $(23.83 \pm 2.02,20.33 \pm 1.25)$. The results do not show a significant relationship between the variables of body composition and the physical tests performed.

Keywords: Talents, Optimist class, anthropometry, body composition, sailor.

\section{Introducción}

La vela está conformada, por muy diferentes clases o tipos de embarcaciones, motivo por el cual, el regatista transita por varias de ellas desde su etapa inicial, hasta elegir la más adecuada y que mejor se ajuste tanto a sus características morfológicas, psicológicas, como de habilidad, conocimiento o motivación.

Un proceso de selección de talentos adecuado para un deporte como la vela, debe tener en cuenta múltiples factores, ya que practican en un medio de gran dureza y variabilidad que hace aún más complicada la intervención. Pero debido a las características tan particulares que posee, la mayoría de los estudios realizados hasta el momento han estado centrados en aquellos deportistas de clases olímpicas, que poco tienen que ver con los que se inician en las clases infantiles, para los que los resultados y conclusiones de estos trabajos, tienen una difícil y compleja aplicación.

\begin{tabular}{|llll|}
\hline Dirección para correspondencia & [Correspodence & address]: \\
Virginia Tejada-Medina. Universidad de Granada. & (España). \\
E-mail: vtejada@ugr.es & & & \\
\hline
\end{tabular}
tos, debemos empezar por concretar los criterios de selección más adecuados, que son los componentes que caracterizan al talento deportivo (Mateo, 1990). Analizar cuáles de esos indicadores son condicionantes del rendimiento en la vela ligera, nos lleva a establecer una relación entre el físico del individuo, la modalidad deportiva practicada y el papel de la constitución física como factor de aptitud deportiva. Como apuntan Sánchez-Muñoz, Requena y Zabala (2003), existe un claro prototipo físico para lograr un óptimo rendimiento a un alto nivel deportivo.

El Optimist es un barco que se encuentra dentro del grupo de los monocascos individuales (Tabla 1. Características técnicas del Optimist).
Para elaborar un programa eficaz de búsqueda de talen- 
Tabla 1. Características técnicas del Optimist.

\begin{tabular}{lcl}
\hline Medidas & & Rectangular \\
\hline Tipo de casco & Uno \\
No de tripulantes & $2,30 \mathrm{~m}$. \\
Eslora total & $2,15 \mathrm{~m}$. \\
Eslora en flotación & $3,50 \mathrm{~m}$. \\
Superficie vélica & 1 \\
No de velas & $35 \mathrm{~kg}$. \\
Peso del casco (mínimo) & $45 \mathrm{~kg}$. \\
Peso total & &
\end{tabular}

Es una clase predominante en la vela ligera infantil, en la que se inician la mayoría de los regatistas y fundamental en la competición infantil hasta los 14 años. Un total de 120 Optimist participan en los Campeonatos y Copas de España cada ańo, tras haber pasado una larga y dura selección en cada autonomía para ganarse el derecho a participar (TejadaMedina, 2012).

Por todo ello, el objetivo del presente estudio es valorar la condición física y definir el perfil antropométrico, los parámetros relacionados con la composición corporal en jóvenes regatistas de la clase Optimist de la Ciudad Autónoma de Melilla, con el interés por conocer cuáles serían los indicadores que podrían justificar el descubrimiento de un talento deportivo en vela ligera.

\section{Material y Método}

El presente trabajo, corresponde con un estudio de casos, de carácter descriptivo y transversal. Se llevó a cabo en la Ciudad Autónoma de Melilla. Fueron estudiados 3 regatistas de la clase Optimist, que ocupan actualmente los primeros puestos del ranking provincial. Las características de los deportistas se muestran en la tabla 2.

Para la valoración de la composición corporal se determinaron los parámetros de peso, talla, pliegues cutáneos (tríceps, suprailíaco, muslo anterior y medial de la pierna) y perímetros musculares (brazo contraído, abdomen, muslo medial y pierna). Se utilizaron una serie de aparatos de uso tradicional entre los estudios de valoración de datos antropométricos: Estadiómetro móvil SECA 217, báscula digital QUICK TANITA TBF 300 GS, cinta antropométrica de acero flexible LUFKIN W606, Plicómetro HOLTAIN con precisión de $0,2 \mathrm{~mm}$., cajón antropométrico de altura regulable sin respaldo y lápiz dermográfico para seńalar los puntos anatómicos y las referencias antropométricas.
Para determinar la composición corporal y por tanto el análisis de los cuatro componentes corporales, se utilizó la metodología propuesta por De Rose y Guimaraes (1980), basada en el modelo tetracompartimental de Matiegka (1921), sustituyendo la fórmula para el cálculo del componente graso por la propuesta de Lohman, Slaughter, Boileau, Bunt, y Lussier (1986) y Lohman (1989) para menores de 15 años.

En cuanto a las pruebas físicas, para evaluar la potencia del tren inferior, se realizaron tres saltos verticales (SJ) sobre la plataforma de contacto (Bosco, 1994), con un intervalo de un minuto entre ellos, anotando el valor más alto alcanzado en centímetros. Los sujetos, se posicionaron sobre la plataforma, con los miembros inferiores en extensión y las manos a la altura de la cintura pélvica para evitar el uso de los miembros superiores durante el salto, y poder alcanzar la máxima altura posible. Para valorar la flexibilidad, desde la posición en bipedestación, se realizó el test de flexión profunda de tronco, anotando el mejor de los tres intentos. Por último, valoramos la fuerza de prensión manual con ambas manos, utilizando el dinamómetro digital TKK5401, realizando dos intentos consecutivos con cada mano y anotando el mejor resultado obtenido con cada una. Previo a la realización de esta prueba, se llevó a cabo una adaptación al instrumento, con una serie de instrucciones verbales sobre el funcionamiento del aparato, acompañadas de un intento de prueba por parte de los sujetos.

Los test se realizaron en 1 día, repartidos en sesión de mañana, para la valoración de la composición corporal, y sesión de tarde para el resto de pruebas físicas.

Para el tratamiento estadístico de los datos se utilizó el software informático SPSS 20.0 para las distintas técnicas estadísticas. Se realizó un análisis descriptivo básico de los datos obtenidos (media \pm desviación típica), una comparación de medias entre las variables en función de la distribución de la normalidad, utilizando la $\mathrm{T}$ de Student para pruebas paramétricas independientes (por el tamaño reducido de la 
muestra, no fue necesario aplicar la prueba de normalidad), y un análisis correlacional.

\section{Resultados}

Las características generales de los sujetos del estudio, están reflejadas en la tabla 2. Los valores descriptivos resultantes de la evaluación de la composición corporal de los sujetos y los registros correspondientes a los tejidos corporales medidos (pliegues y perímetros musculares), se muestran en las tablas 3 y 4 .

Tabla 2. Características de los participantes.

\begin{tabular}{lcccc}
\hline & Sujeto 1 & Sujeto 2 & Sujeto 3 & Total \\
\hline & & & & $\mathrm{M} \pm \mathrm{DT}$ \\
\hline Edad (años) & 12,9 & 11,7 & 13,9 & $12,8(1,1)$ \\
Talla $(\mathbf{m})$ & 149 & 147,8 & 152 & $149,6(2,16)$ \\
Peso $(\mathbf{k g})$ & 37,2 & 38,20 & 31,4 & $35,6(3,67)$ \\
IMC $\left(\mathbf{K g} / \mathbf{m}^{2}\right)$ & 16,8 & 17,40 & 13,60 & $15,93(2,04)$ \\
\hline
\end{tabular}

Tabla 3. Composición corporal.

\begin{tabular}{lcccc}
\hline & Sujeto 1 & Sujeto 2 & Sujeto 3 & Total \\
\hline \% Grasa & & & & $\mathrm{M} \pm \mathrm{DT}$ \\
Masa Grasa & 3,20 & 11,20 & 4,20 & $7,86 \pm 3,51$ \\
Masa Magra & 34,10 & 4,30 & 1,30 & $2,90 \pm 1,50$ \\
Agua total & 25,00 & 24,80 & 30,10 & $32,70 \pm 2,25$ \\
\hline
\end{tabular}

Tabla 4. Valores de las medidas de pliegues y perímetros musculares.

\begin{tabular}{lcccc}
\hline & Sujeto 1 & Sujeto 2 & Sujeto 3 & Total \\
\hline Tríceps & & & & $\mathrm{M} \pm \mathrm{DT}$ \\
Suprailíaco & 12,00 & 15,00 & 13,00 & $13,33 \pm 1,52$ \\
Muslo anterior & 24,00 & 7,00 & 5,00 & $5,66 \pm 1,15$ \\
Medial pierna & 11,00 & 20,00 & 17,00 & $20,33 \pm 3,51$ \\
E Pliegues & 52,00 & 57,00 & 49,00 & $52,66 \pm 4,04$ \\
P. Brazo contraído & 22,50 & 24,00 & 19,30 & $21,93 \pm 2,40$ \\
P. Abdominal & 65,00 & 63,00 & 57,50 & $61,83 \pm 3,88$ \\
P. Muslo medial & 42,50 & 43,00 & 36,50 & $40,66 \pm 3,61$ \\
P. Pierna & 29,00 & 31,00 & 25,50 & $28,50 \pm 2,78$ \\
\hline
\end{tabular}

En la tabla 5 se presentan los resultados de la valoración funcional de los sujetos, SJ (altura del CDG y tiempo de vuelo), flexibilidad y dinamometría manual (derecha e izquierda).
Tabla 5. Valoración funcional.

\begin{tabular}{lcccc}
\hline & Sujeto & Sujeto & Sujeto & \multirow{2}{*}{ Total } \\
\hline & $\mathbf{1}$ & $\mathbf{2}$ & $\mathbf{3}$ & \\
\hline SJ HCDG (cm) & 20,60 & 23,70 & 23,30 & $22,53 \pm 1,68$ \\
SJ TV (sg) & 410 & 440 & 436 & $428,6 \pm 16,28$ \\
Flexibilidad (cm) & 30,00 & 30,00 & 32,00 & $31,67 \pm 1,15$ \\
Dinamometría D (Kg) & 22,00 & 26,00 & 23,50 & $23,83 \pm 2,02$ \\
Dinamometría I (Kg) & 20,50 & 21,50 & 19,00 & $20,33 \pm 1.25$ \\
\hline
\end{tabular}

De lo expuesto anteriormente en los valores descriptivos, podemos extraer que el sujeto 2 , siendo el de menor edad y talla, obtiene unos valores superiores a la media en todas las pruebas de valoración funcional. En lo referente a la composición corporal, este mismo sujeto supera a la media en el porcentaje de grasa corporal $(11,2 \%)$, datos que explican los valores obtenidos en el sumatorio de pliegues y medidas perimetrales, superiores al resto de regatistas y a la media total del grupo.

El estudio de homogeneidad de medias para muestras apareadas ( $\mathrm{T}$ de Student), presenta diferencias significativas $(\mathrm{p}<0,001)$ entre la talla media de los regatistas y algunas de las variables estudiadas como la fuerza de prensión manual con ambas manos, el salto vertical, la flexibilidad y los datos de composición corporal. Por otra parte, las variables peso e IMC han presentado diferencias significativas con el tiempo de vuelo en el salto vertical. En la tabla 6 se muestran los resultados más significativos.

Tabla 6. Comparación de medias.

\begin{tabular}{|c|c|c|}
\hline & \multicolumn{2}{|c|}{ Talla } \\
\hline & T de Student & Valor de $\mathrm{p}$ \\
\hline SJ HCDG $(\mathrm{cm})$ & 85,51 & $<0,000$ \\
\hline SJ TV (sg) & $-29,88$ & $<0,001$ \\
\hline Flexibilidad $(\mathrm{cm})$ & 187,01 & $<0,000$ \\
\hline Dinamometría D $(\mathrm{Kg})$ & 61,95 & $<0,000$ \\
\hline Dinamometría I (Kg) & 65,56 & $<0,001$ \\
\hline$\sum$ Pliegues & 27,52 & $<0,001$ \\
\hline
\end{tabular}

El análisis correlacional se ha realizado atendiendo al agrupamiento de las variables talla, peso e IMC con el resto de las variables. Los resultados no presentan una correlación significativa. Por otra parte, en el análisis correlacional llevado a cabo entre todas las variables, se aprecia una correlación significativa entre el SJ (HCG) y la masa magra; y tendencia a la significación $(\mathrm{p}<0,005)$, entre la dinamometría manual izquierda, el porcentaje de grasa corporal y el perímetro de la pierna. 


\section{Discusión}

De todos es sabido que los deportistas que participan en diferentes disciplinas deportivas difieren en sus características físicas o morfológicas, de forma que, para obtener óptimos resultados, el entrenamiento debe estar basado en estos parámetros diferenciales. En vela, el éxito deportivo puede estar influido, entre otros factores, por la elección correcta del barco en el que se quiere competir, ya que al margen de la incertidumbre creada por el propio entorno en el que se desarrolla la regata, un perfil antropométrico o físico determinado, puede predisponer al regatista hacia una clase u otra.

Una de las grandes preocupaciones que ha venido siendo objeto de estudio en numerosas investigaciones en los últimos años, es saber si existe o no un patrón funcional o antropométrico específico de cualquier deportista profesional. Aunque se ha aceptado que la congruencia entre los somatotipos de un mismo deporte, se suele alcanzar cuanto mayor es el nivel de rendimiento, la última tendencia apunta hacia el análisis de los somatotipos antes de llegar a estos estadíos de alto rendimiento, para poder seleccionar a tiempo un posible talento.

Teniendo en cuenta los resultados derivados de este estudio, consideramos en primer lugar, que los datos de talla $(1.50-1.60 \mathrm{~m})$ y peso $(45-55 \mathrm{Kg})$ aportados por la Federación Española de Vela en Renom-Pinsach y Violán-Corominas (2002), en cuanto a las características físicas de los regatistas de Optimist, nos permiten comparar con los sujetos objeto de esta investigación, comprobando que éstos, se encuentran por debajo de las medias establecidas en lo que respecta a estas dos variables.

Comparando los valores de peso y talla obtenidos en nuestro estudio con los encontrados en los trabajos realizados por Alcaraz, Martínez, Carrasco y Navarro (2008),
Palomino-Martín, Quintana-Santana, Quiroga-Escudero y González-Muñoz (2017), Oliveira, Polato, Alves, Frega y Macedo (2011) y Tejada-Medina (2012), con regatistas de la clase infantil Optimist, observamos grandes diferencias en estas dos variables, estando por debajo los sujetos estudiados en nuestra investigación. Esto puede deberse a que la edad media de los sujetos estudiados $(12,8 \pm 1,1)$ es inferior a las de los demás regatistas, por tanto, los de mayor edad tienden a ser los más altos y pesados.

Como fenómeno curioso, cabe destacar, que la organización reguladora de esta clase, la International Optimist Dinghy Association (IODA, 2009), ha constatado que las dimensiones del Optimist ya no se ajustan tan bien a las características antropométricas de sus tripulantes. La talla y el peso de éstos han aumentado considerablemente, en relación con los valores que eran considerados hace ańos como normales para las edades de la banda superior de la clase. Esto ha repercutido en las prestaciones de la embarcación, la calidad de la navegación y en el momento en que se plantea el cambio hacia una clase superior.

En cuanto al IMC, los datos extraídos de nuestro estudio, sitúan a los regatistas infantiles en el límite entre las categorías de bajo-peso y normo-peso, ya que según los valores generales de los patrones de crecimiento propuestos por la OMS (Wilson, 2005) para niños y adolescentes, los valores por debajo del percentil 5, corresponden con los sujetos con bajo-peso, y con peso normal, aquellos que se encuentren dentro del rango 5-85. Los estudios llevados a cabo por Tejada-Medina (2012), Oliveira et al. (2011) y Alcaraz et al. (2008), muestran valores de 18,27 $\pm 1,7,19,6 \pm 2,8,18,7 \pm 2,2$ y $18,4 \pm 1,0$ respectivamente. Todos estos datos están reflejados en la tabla 7 .

Tabla 7. Comparación de los valores de peso, talla e IMC.

\begin{tabular}{|c|c|c|c|c|}
\hline \multirow{2}{*}{ Autores } & Edad & Peso & Talla & IMC \\
\hline & $\mathrm{M} \pm \mathrm{DT}$ & $\mathrm{M} \pm \mathrm{DT}$ & $\mathrm{M} \pm \mathrm{DT}$ & $\mathrm{M} \pm \mathrm{DT}$ \\
\hline Sujetos de nuestro estudio & $12,8 \pm 1,1$ & $35,6 \pm 3,67$ & $149,6 \pm 2,16$ & $15,93 \pm 2,04$ \\
\hline $\begin{array}{l}\text { Palomino-Martín, A., Quintana-Santana, D., Quiroga-Escudero, M.E. \& } \\
\text { González-muñoz, A. (2017) }\end{array}$ & $14 \pm 0,77$ & $48,8 \pm 6,37$ & $160 \pm 5,4$ & \\
\hline $\begin{array}{l}\text { Palomino-Martín, A., Quintana-Santana, D., Quiroga-Escudero, M.E. \& } \\
\text { González-muñoz, A. (2017) }\end{array}$ & $13,2 \pm 1,1$ & $44,2 \pm 7,1$ & $155,6 \pm 8$ & \\
\hline Tejada-Medina, V. (2012) & $13,13 \pm 1,28$ & $45,75 \pm 8,32$ & $157 \pm 10,9$ & $18,27 \pm 1,7$ \\
\hline Oliveira, L.F., Polato, D., Alves, R., Frega, S. \& Macedo, A. (2011) & $11-12$ & $45,6 \pm 5,5$ & $152,8 \pm 5,8$ & $19,6 \pm 2,8$ \\
\hline Oliveira, L.F., Polato, D., Alves, R., Frega, S. \& Macedo, A. (2011) & $13-14$ & $48,9 \pm 7,5$ & $161,7 \pm 7,7$ & $18,7 \pm 2,2$ \\
\hline Alcaraz, P., Martínez, E., Carrasco, L. \& Navarro, F.J. (2008) & $13,4 \pm 1,2$ & $44, \pm 6,9$ & $155,5 \pm 11,0$ & $18,4 \pm 1,0$ \\
\hline Tan, B. \& Sunarja, F. (2007) & $11,6 \pm 1,4$ & $37,2 \pm 8,2$ & & \\
\hline
\end{tabular}

Por otro lado, un aspecto considerado en la mayoría de las investigaciones con jóvenes y adolescentes, es el porcenta- je de grasa corporal. En los estudios previos realizados con regatistas de clase Optimist (Oliveira et al., 2011), se deter- 
minó el \%MG en 17 regatistas de categoría infantil (11-12 años) y 33 juveniles (13-14 años), obteniéndose diferencias entre ambas categorías, siendo inferiores los de mayor edad $(18,6 \pm 5,5)$, frente a los infantiles $(23,9 \pm 7,0)$. Estos datos difieren de los obtenidos por Tejada-Medina (2012) con 97 regatistas $(13,13 \pm 1,28$ años) para los que el \%MG superó los 14 puntos, 13,3 $\pm 3,9$. Comparando nuestros datos con los de ambos estudios, encontramos valores muy bajos en los sujetos evaluados $(7,86 \pm 3,51)$, que se alejan bastante de los valores medios aportados anteriormente.

Atendiendo a los pliegues y perímetros medidos, aparecen valores significativamente mayores en los pliegues del tríceps, muslo y pierna para nuestros sujetos, en relación a los datos aportados por Palomino-Martín et al. (2017) con un grupo de 158 regatistas de Optimist participantes en un campeonato del mundo, divididos en "grupo Top" y "grupo Fleet" (Top: 9,5 $\pm 2,9,12,8 \pm 4,5,12,5 \pm 4,5$; Fleet: 10,5 $\pm 3,6,14,1 \pm 5,2$, $12,6 \pm 4,5)$. Sólo se obtuvieron valores inferiores para nuestros sujetos en el pliegue suprailíaco frente a los grupos anteriormente citados (Top: 6,5 $\pm 3,2$; Fleet: 7,4 $\pm 3,6$ ).

Los valores de los perímetros del brazo contraído, muslo y pierna, fueron significativamente inferiores a los presentados por Tejada-Medina (2012), con datos de 23,16 $\pm 2,39$, $43,19 \pm 4,31$ y $31,61 \pm 2,64$ respectivamente.

En cuanto a la fuerza de prensión manual y el salto vertical, los datos extraídos de nuestro estudio, muestran valores significativamente inferiores respecto a los resultados obtenidos por Oliveira, et al., (2011) y Alcaraz, et al. (2008) en el SJ para la categoría juvenil $(31,6 \pm 4,9)$ en el primero, y el grupo de 8 optimistas $(34,4 \pm 5,5)$ en el segundo. La fuerza de prensión manual sólo fue estudiada por Oliveira, et al. (2011) sobre la mano dominante de los regatistas, dicha investigación presenta valores superiores en la categoría juvenil $(29,6 \pm 5,5)$, y ligeramente inferiores en la infantil $(22,5 \pm 2,9)$.
Por último, en la prueba de flexibilidad de tronco, los sujetos objeto de estudio mostraron valores superiores, frente a lo aportados por Alcaraz, et al. (2008) $(20,3 \pm 8,2)$.

\section{Conclusiones}

En los diversos apartados de esta investigación se han ido presentando y analizando aquellas aportaciones más significativas e importantes, con el fin de ayudarnos a conocer y comprender las particularidades de esta modalidad de la vela ligera, así como los indicadores para establecer perfiles antropométricos o físicos que nos permitan reconocer un talento deportivo.

Respecto a los resultados derivados del proceso de investigación, consideramos que los datos de composición corporal obtenidos, muestran niveles muy bajos para todas las variables en relación a los valores medios encontrados en diversas investigaciones llevadas a cabo con regatistas de la misma clase. Para la valoración antropométrica, sólo el pliegue suprailíaco presenta valores inferiores al resto de regatistas estudiados.

Las pruebas físicas de SJ, dinamometría y flexibilidad muestran una variabilidad en los datos, siendo inferiores los referentes al salto vertical, no así los obtenidos para flexibilidad y fuerza de prensión manual con regatistas de edades similares.

Por tanto, tras los resultados obtenidos, podemos afirmar que los regatistas estudiados no presentan un perfil antropométrico cercano a los patrones establecidos en otras investigaciones realizadas. En cuanto a los valores para fuerza manual y flexibilidad, debemos decir que a pesar de ser superiores a los estudios con los que han sido comparados, son pocos los autores que han estudiado estas capacidades en la vela ligera, y concretamente en la clase Optimist.

\section{Referencias}

1. Alcaraz, P.E., Martínez, E., Carrasco, L., y Navarro, F.J. (2008). Condición física, perfil antropométrico y composición corporal en jóvenes regatistas de alto nivel. Tándem, 28, 76-84.

2. Bosco, C. (1994). La valoración de la fuerza con el test de Bosco. Barcelona. Editorial Paidotribo.

3. De Rose, E.H., y Guimaraes, A.C. (1980). Model for optimization of somatotype in Young athletes. In M. Ostyn, G. Beunen y J. Simons (Eds.), Kinanthropometry II. Baltimore: Unversity Park Press.

4. International Optimist Dinghy Association (2009). The Optimist class in the world. Recuperado 7 de Agosto, 2009, de http://www.optiworld. org/basics.html\#world.

5. Mateo, J. (1990). La Batería Eurofit como medio de detección e talentos. Apunts: Educación Física y Deportes, 22, 59-68.

6. Matiegka, J. (1921). The testingof physical efficiency. American Journal of Physical Anthropology, 4, 223-230.

7. Lohman, T.G. (1989). Assesment of body composition in children. Pediatric Exercise Science, 1(1), 19-30.

8. Lohman, T.G., Slaughter, M.H., Boileau, R.A., Bunt, J., y Lussier, L.
(1986). Applicability of body composition techniques and constants for children and youths. Exercise and Sport Sciences Reviews, 14(1). $352-$ 358.

9. Oliveira, L., Polato, D., Alves, R., Fraga, S., y Macedo, A. (2011). Perfil antropométrico e funcional de velejadores da classe "Optimist". Revista Brasileira de Educaçấo Física e Esporte, 25(1), 173-179. https://dx.doi. org/10.1590/S1807-55092011000100016.

10. Palomino-Martín, A., Quintana-Santana, D., Quiroga-Escudero, M.E. y González-Muñoz, A. (2017). Incidence of anthropometric variables on the performance of top Optimist sailors. Journal of Human Sport and Exercise, 12(1), 41-57. doi: 10.14198/jhse.2017.121.04.

11. Renom, J. y Violán, J.A. (2002). Entrenamiento psicológico en vela (1a ed.). Barcelona: Editorial Paidotribo.

12. Sánchez-Muñoz, C., Requena, B., y Zabala, M. (2003). Determinación del perfil antropométrico de jóvenes corredores de medio fondo de élite. Lecturas: Educación Física y Deportes. Revista Digital, 8(58). Recuperado de http://www.efdeportes.com/efd58/mediof.htm.

13. Tan, B., y Sujarna, F. (2007). Body mass changes and nutrient intake 
of Optimist class sailors on a race day. Journal of Sports Science, 25(10), 1137-1140.

14. Tejada-Medina, V. (2012). Análisis de las variables antropométricas y su influencia sobre el rendimiento deportivo en regatistas de la clase Optimist de vela. Granada: Editorial Universidad de Granada.

15. Wilson, S. (2005). How Body Mass Index Works. HowStuffWorks. com. Recuperado de http://health.howstuffworks.com/bmi.htm 